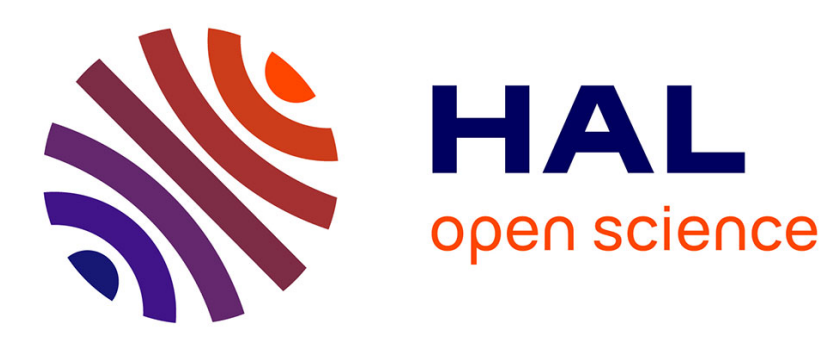

\title{
Breakage of non-Newtonian character in flow through a porous medium: Evidence from numerical simulation.
}

Jérémy Bleyer, Philippe Coussot

\section{To cite this version:}

Jérémy Bleyer, Philippe Coussot. Breakage of non-Newtonian character in flow through a porous medium: Evidence from numerical simulation.. Physical Review E : Statistical, Nonlinear, and Soft Matter Physics, 2014, 89 (6), pp.063018. 10.1103/PhysRevE.89.063018 . hal-01055574

\section{HAL Id: hal-01055574 \\ https://hal-enpc.archives-ouvertes.fr/hal-01055574}

Submitted on 25 May 2015

HAL is a multi-disciplinary open access archive for the deposit and dissemination of scientific research documents, whether they are published or not. The documents may come from teaching and research institutions in France or abroad, or from public or private research centers.
L'archive ouverte pluridisciplinaire HAL, est destinée au dépôt et à la diffusion de documents scientifiques de niveau recherche, publiés ou non, émanant des établissements d'enseignement et de recherche français ou étrangers, des laboratoires publics ou privés. 


\title{
Breakage of non-Newtonian character in flow through porous medium: evidence from numerical simulation
}

\author{
J. Bleyer, P. Coussot \\ Laboratoire Navier, Univ. Paris-Est, Champs sur Marne, France
}

\begin{abstract}
We study the flow, through a model 2D porous medium, of Newtonian fluids, powerlaw fluids and viscoplastic fluids in the laminar regime and with moderate or dominant effects of the yielding term. A numerical technique able to take properly into account yielding effects in viscoplastic flows without any regularization is used to determine the detailed flow characteristics. We show that as soon as the distance between the disks forming the porous medium is sufficiently small, the velocity field, and in particular the distribution function of the velocity of these different fluids in a wide range of flow regimes are similar. Moreover the volume fraction of fluid at rest is negligible even at low flow rate. Thus the non-Newtonian character of a fluid flowing through such a complex geometry tends to be broken. We suggest that this is due to the fact that in a flow through a channel of rapidly varying cross-section, the deformation, and thus the flow field, is imposed to the fluid, a situation which is encountered almost everywhere in a porous medium. These results make it possible to deduce a general expression for the Darcy's law of these fluid types and estimate the parameters appearing in this expression.
\end{abstract}

\section{Introduction}

Flows of non-Newtonian fluids are involved in a wide range of applications [1] such as the injection of cement in soils, penetration of glue in porous substrates, propagation of blood through kidney, hydraulic fracture [2], etc. One of the most important applications concerns oil reservoir engineering in which a wide range of complex fluids (foams, polymers, emulsions) are used to improve oil recovery [3]. In these processes the flow characteristics of such fluids in porous media remain poorly known due to the complexity of the fluid behaviour and the opacity of the solid structure.

For yield stress fluids (mud, cement, concentrated emulsions or foams), which flow like liquids only beyond a critical stress and behave as solids otherwise, various analytical or numerical approaches [4] have been developed in order to predict the pressure gradient $(\nabla p)$ vs average velocity $(\bar{V})$ through the porous medium, but the validity of their physical assumptions could not be checked in the absence of any information concerning the flow characteristics inside the medium. For Newtonian fluids (of constant viscosity $\mu$ ) we know that any element of the liquid network flows and in the absence of inertia effects the velocity field scaled by the average velocity $(\bar{V})$ is constant (Stokes flow). As a consequence $\nabla p$ is proportional to $\bar{V}$ by a factor $\mu / K$ in which $K$ is a characteristic of the porous medium, namely its permeability. Some studies suggested that with yield stress fluids two critical effects could occur: (i) at the pore scale the flowing volume increases with the pressure gradient [5] and (ii) at a macroscopic scale the flow starts as a percolation effect, i.e. at a critical pressure drop liquid regions exist only along a specific path throughout the porous medium [5-6] and as $\nabla p$ is increased more flowing paths progressively form within the porous medium. 
Recent simulations [7] of Bingham fluid flow through porous medium using a LatticeBoltzmann TRT (Two Relaxation Time) scheme support these assumptions: as the pressure increases the fluid flows first in only one channel, then new paths open and finally all the fluid flows.

On the other hand a recent experimental study [8] of the flow characteristics inside a model bead packing provided unexpected results which contradict the above conclusions. An NMR (Nulcear Magnetic Resonance) technique was used, which provided straightforward information on the average local flow characteristics, more precisely the probability density function (pdf) for the velocity, without being affected by any spatial resolution problem. It was shown [8] that the pdf is apparently similar for Newtonian fluids, power-law fluids and viscoplastic fluids in the laminar regime and with moderate or dominant effects of the yielding term. Moreover it appears that the fraction of region of fluid at rest is negligible even at low $\bar{V}$ values. Under these conditions all occurs as if the non-Newtonian character of the fluid was broken.

In the present work we provide a further analysis of this problem and suggestions for understanding it. From numerical simulations using a 2D finite element discretization and a second-order cone programming optimization solvers, which are able to take properly into account yielding effects in viscoplastic flows without any regularization, we show that the same result (i.e. breakage of the non-Newtonian character) is obtained in a simple 2D porous geometry. We suggest that this is due to the fact that in a flow through a channel of rapidly varying cross-section, if the macroscopic flow rate is imposed, the deformation, and thus the flow field, is essentially imposed to the fluid, a situation which is encountered almost everywhere in a porous medium.

\section{Materials, geometry and simulation technique}

In this work we developed specific tools for modelling yield stress fluid flows as this is a complex situation which encompasses simpler cases such as Newtonian or shear-thinning fluid flows.

The constitutive equation of simple yield stress fluids (with negligible thixotropic character) in simple shear can be very well represented by a HB (Herschel-Bulkley) model: $\tau<\tau_{c} \Rightarrow \dot{\gamma}=0$ (solid regime); $\tau>\tau_{c} \Rightarrow \tau=\tau_{c}+k \dot{\gamma}^{n}$ (liquid regime), in which $\tau$ is the shear stress, $\dot{\gamma}$ the shear rate, $\tau_{c}$ the yield stress, and $k$ and $n$ two material parameters [9]. In laminar conditions the flow is governed by the Bingham number, $\mathrm{Bi}=\tau_{c} / k \dot{\gamma}^{n}$, where $\dot{\gamma}$ is a characteristic shear rate which can be written as the ratio of a characteristic velocity $(V)$ to a characteristic length $(l)$ of the system. This dimensionless number estimates the ratio of the constant (plastic) to the ratedependent (viscous) parts of the constitutive equation, and finally gives an idea of the relative importance of the solid and liquid regions in the sample. Thus flows through similar porous media at different average velocities and with different pore sizes (similar distributions when rescaled by a characteristic length) or yield stress values have similar flow characteristics (after appropriate rescaling using the characteristic length and velocity) as soon as they have the same Bingham number. Varying $\mathrm{Bi}$ in a wide range makes it possible to include almost purely plastic flows ( $\mathrm{Bi}>>1)$, flows with both plastic and viscous effects playing a significant role $(\mathrm{Bi} \approx 1)$, simple power-law fluid flows $(\mathrm{Bi}=0)$. We also did simulations for Newtonian fluids $(\mathrm{Bi}=0$ and $n=1)$. 
Since the effects above described concerning the pdf have been observed in a specific porous medium, namely a granular packing, we have first to discuss their generality for any porous medium. We can consider that a typical porous medium is basically made of (possibly interconnected) channels in which the fluid can flow throughout the system. In general the crosssection of these channels widely varies (from one "pore" to another) and they are not straight (tortuosity). The geometry of a granular packing effectively contains these basic ingredients.

However numerical simulations of flows through a 3D bead packing pose several problems: it is difficult to make precise simulations in a volume including more than a few beads, due to the high computational cost of adopting a very dense $3 \mathrm{D}$ mesh to properly describe the porous geometry as well as the potential interfaces in the fluid domain between flowing and arrested regions in the case of yield stress fluids; it is rather uneasy to compare the local flow characteristics (in the form of 3D velocity fields) obtained when varying some parameters, so that people generally tend to focus on possible preferential flow regions or macroscopic properties (i.e. permeability) [10], but we can fear that in a disordered system observed at a relatively small scale these characteristics are greatly influenced by the specificities of the geometrical region under consideration.

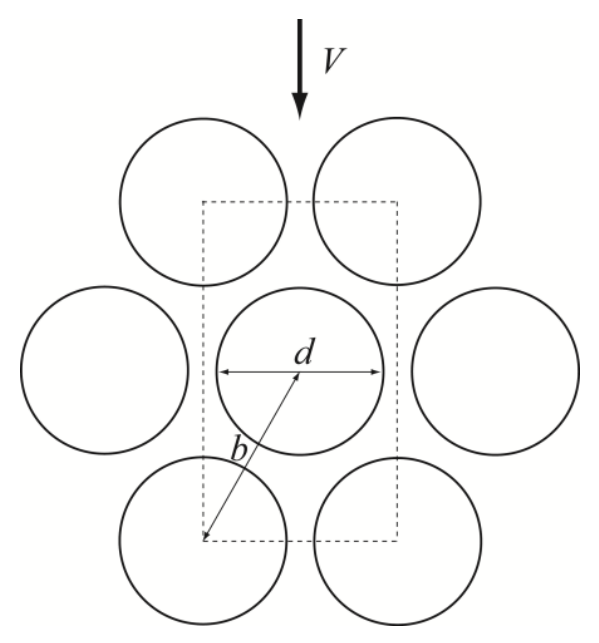

Figure 1: Scheme of the flow geometry.

In order to avoid these problems and in particular obtain readily comparable data for different fluid types under different flow regimes we carried out 2D numerical simulations of flow through an ordered array of disks (which may be seen in 3D as cylinders perpendicular to the plane of observation). More precisely the geometry consists in identical circles of diameter $d$ situated at equal distance $b$ (between centers) from each other (see Figure 1). By this way we represent interconnected tortuous channels of rapidly varying cross-sections and we avoid the problem of preferential flows induced by heterogeneities observed at a local scale. Moreover the velocity fields can now be studied and compared in detail. Note that it may be considered that an apparent strong difference between such a geometry and a real 3D bead packing is the absence of contacts between the disks. Actually a 2D image of the section of a bead packing is a similar picture, namely a set of disks of various sizes dispersed in the plane with only a very small fraction of them in contact (this fraction increases with the thickness of the section). This means that in a $2 \mathrm{D}$ planar section through the bead packing the flow can be considered to have 
general trends close to that through our set of similar disks except that there are additional flows entering or leaving this section due to the $3 \mathrm{D}$ structure.

The simulations are carried out assuming a similar flow field in each similar area of this periodic array. The only imposed condition is a constant average velocity $V$ through the porosity ( $V=\bar{V} / \varepsilon$, where $\varepsilon$ is the porosity, which here expresses as $\left.\varepsilon=1-(\pi / 2 \sqrt{3})(d / b)^{2}\right)$. Here we will focus on the velocity fields for different values of the ratio $b / d$, namely $10 / 6,10 / 8$, and 10/9, which correspond to a minimum relative distance between solid surfaces $(a=(b-d) / d)$ equal to $2 / 3,1 / 4$, and $1 / 9$. This range thus encompasses situations for which the particles are at a distance from the order of their size to situations for which they are at a distance much smaller than their size.

Simulation of yield stress fluid flows is a challenging task from a numerical point of view due to the non-regular nature of the constitutive relation between stresses and strain rates. A simple approach, which can be classified as "regularizing methods", consists of approximating the visco-plastic behavior by a more regular constitutive relationship, generally involving a socalled regularizing parameter. The regularizing model consists in assuming that at low shear rates the material exhibits a high viscosity (e.g. bi-viscous model, Papanastasiou model) [11]. However, the distinction between rigid and flowing region is much harder since rigid regions are approximated as liquid regions flowing at a very small rate. Besides, the solution process can deteriorate when changing the regularization parameter. It is not clear whether such a technique can provide relevant results in the case of complex flows.

The other category of numerical methods, namely "non-regularizing methods", aims at solving the original non-smooth visco-plastic problem using a variational formulation and efficient optimization techniques. The present work falls into this category so as to keep the true viscoplastic nature of the yield stress fluid without introducing any artificial parameter. It uses a second-order cone programming (SOCP) formulation of the arising minimization problem, for which dedicated interior point solvers are available. It has been suggested quite recently [12] that such state-of-the-art numerical tools are extremely efficient for the simulation of yield stress fluid flows and offer some computational advantages over traditional techniques like the Augmented Lagrangian approach for instance [13].

More precisely, the simulations rely on a finite element discretization of the 2D fluid domain using a quadratic interpolation of the velocity field inside each triangular element. The energy minimum principle is then discretized and the minimization problem is reformulated in a form suitable for by the SOCP solver Mosek [14]. An interesting feature is that the strain compatibility and incompressibility equations are directly enforced at the element level so that there is no need to introduce any auxiliary mechanical fields as Lagrange multipliers. Besides, the optimization solver is tailored so that there is no need to tune any algorithmic input parameter. More details on the numerical implementation and the validation of the technique can be found in [12].

\section{Results}

In Figures 2-4 we present the velocity fields obtained in the Newtonian case $(n=1)$ and in the case $n=0.4$ for a series of $\mathrm{Bi}$ number values in the range [0-100]. We thus cover the Newtonian 
case, a typical pure shear-thinning case $(n=0.4, \mathrm{Bi}=0)$, a typical Herschel-Bulkley behavior $(n=0.4)$ with comparable effect of the yielding and the shear-dependent terms $(\mathrm{Bi}=1)$, dominant effect of the yielding term $(\mathrm{Bi}=10)$ and almost purely plastic flow $(\mathrm{Bi}=100)$. Here the Bingham number is computed using $V$ for the velocity and $l=b-d$ for the characteristic length.

For $a=2 / 3$, the velocity fields are clearly different (see Figure 2) in the different situations. The contours of the field are similar for the different values of $n$ for $\mathrm{Bi}=0$, except for the highest velocity range. On the contrary these contours significantly evolve for a fixed $\mathrm{n}$ value when $\mathrm{Bi}$ increases from 0 to 100: the region along the mean flow direction separating two neighbouring particles progressively takes a shell shape, the region of almost constant velocity in the diagonal flow channel widens, and the size of the stagnant region (dark blue) above and before a particle increases. For $a=1 / 4$ the same effects are observed (see Figure 3 ) but they are slightly less marked: the region between two particles has already almost a shell shape for $\mathrm{Bi}=0$ and the width of almost constant velocity in the diagonal flow does not vary significantly when Bi increases.
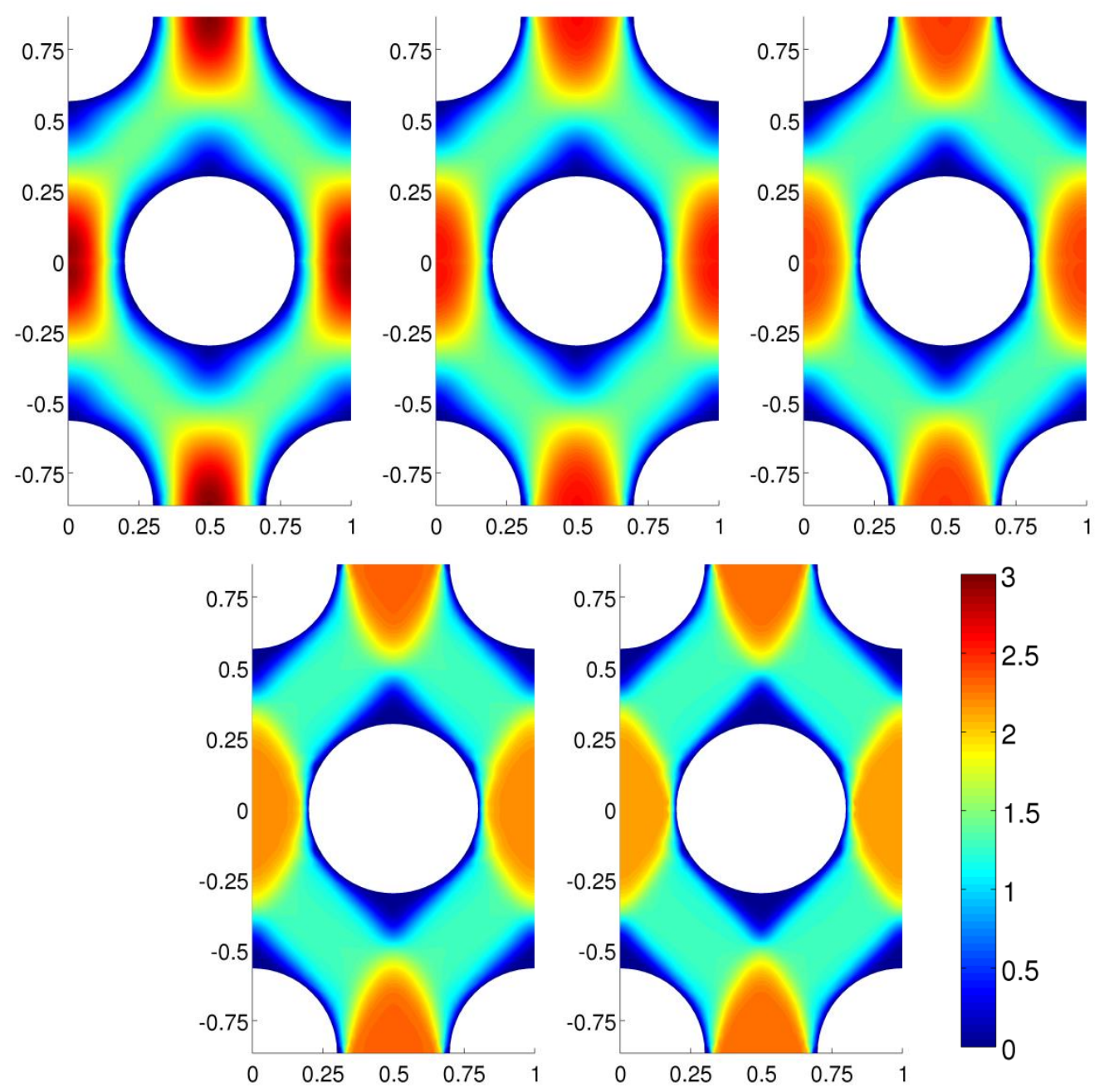

Figure 2: (Color online) Field of the velocity modulus in the model porous medium (here $a=2 / 3$ ) scaled by the average velocity through the void volume $(V)$ and represented in colour scales for different materials and flow regimes: (from left to right) $(n=1) \mathrm{Bi}=0 ;(n=0.4) \mathrm{Bi}=0,1,10,100$. 

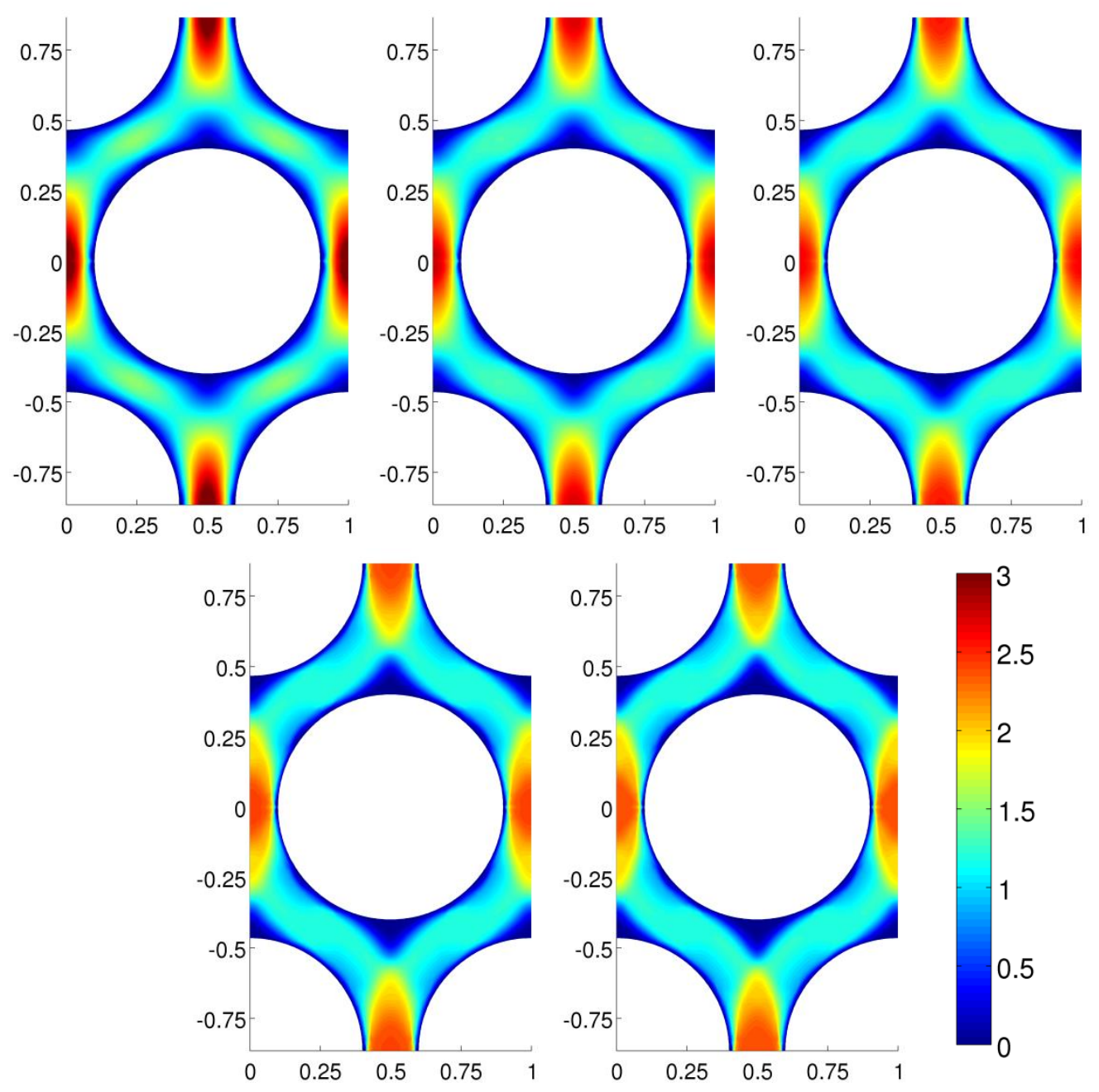

Figure 3: (Color online) Field of the velocity modulus in the model porous medium (here $a=1 / 4$ ) scaled by the average velocity through the void volume $(V)$ and represented in colour scales for different materials and flow regimes: (from left to right) $(n=1) \mathrm{Bi}=0 ;(n=0.4) \mathrm{Bi}=0,1,10,100$.

For $a=1 / 9$ these evolutions are much less marked (see Figure 4): at first sight the velocity fields appear similar. In fact there are still some slight local differences: the stagnant region below and above the particle grows with $\mathrm{Bi}$ and tends to a small cone which approximately represents about $1 / 20$ of the total fluid volume, the extent of the region of almost constant velocity in the diagonal channel decreases with the Bingham number. Besides it is interesting to remark that now the Newtonian case yields a velocity field with a global aspect rather close to those for the shear-thinning or plastic cases. 

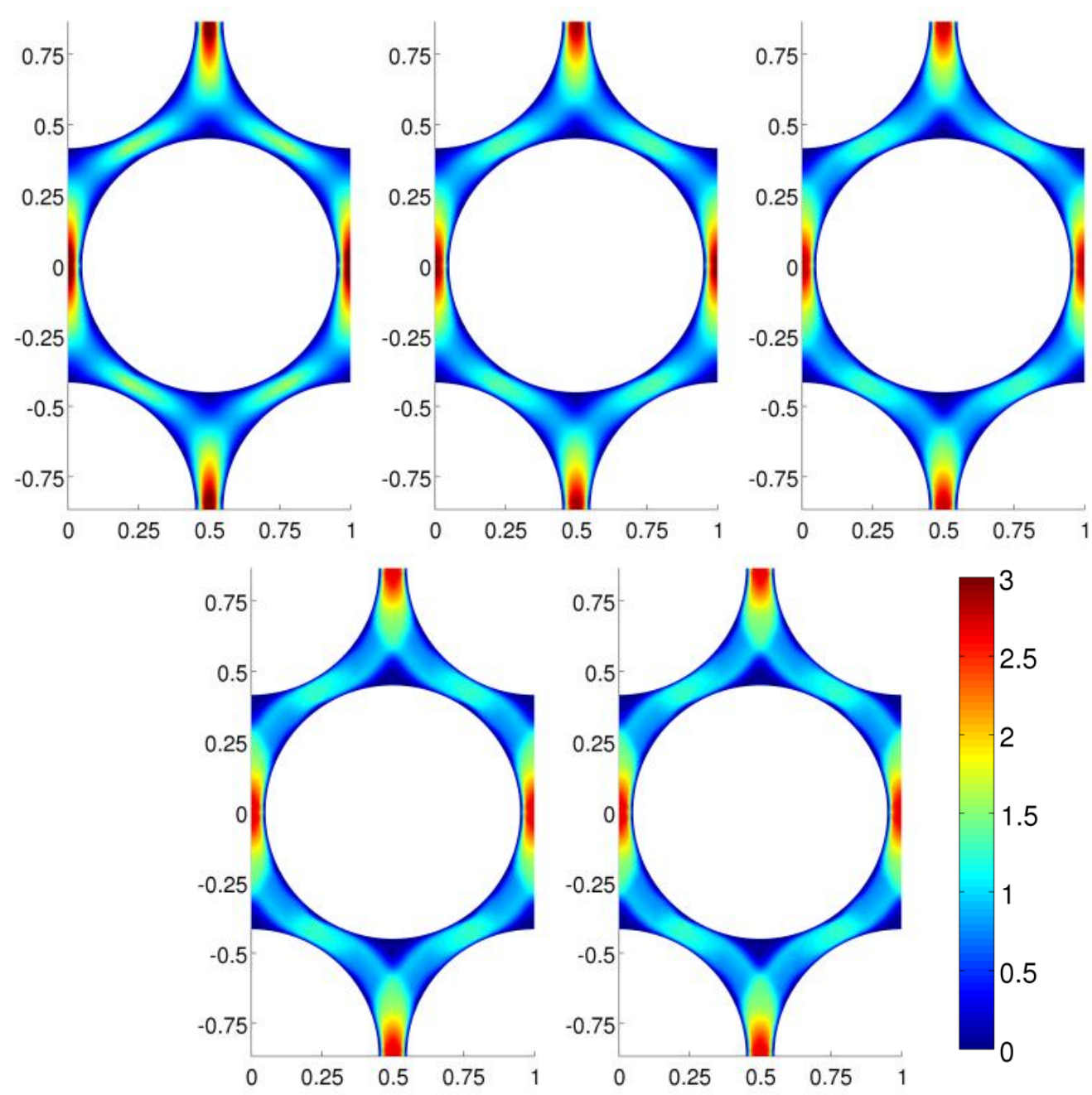

Figure 4: (Color online) Field of the velocity modulus in the model porous medium (here $a=1 / 9$ ) scaled by the average velocity through the void volume $(V)$ and represented in colour scales for different materials and flow regimes: (from left to right) $(n=1) \mathrm{Bi}=0 ;(n=0.4) \mathrm{Bi}=0,1,10,100$.

It finally appears that the velocity fields observed for the different fluids and under different flow regimes exhibit differences which for most of them tend to disappear when $a$ tends to 0 . In order to more precisely appreciate this effect it is necessary to have a quantified characterization of the velocity fields.

In that aim we can compute the pdf for each of the two components of the velocity (vertical and transversal). This pdf, often called "1D velocity propagator", and which is noted $f$, is such that the fraction of fluid having a velocity between $v$ and $v+\mathrm{d} v$ is $f(v) \mathrm{d} v$. In order to appreciate the data in a straightforward way we think it more appropriate to look at the distribution function defined as: $F(v)=\int_{-\infty}^{v} f(u) \mathrm{d} u$. For example, for the flow through a capillary, $F$ is a straight line between 0 and the maximum velocity. For a yield stress fluid there is an unsheared region (plug) around the center of the duct which is associated with a vertical jump at the end of the distribution (i.e. for the maximum velocity) [8]. The height of this step varies from 0 to 1 when $\mathrm{Bi}$ varies from 0 to infinity, so that in the latter extreme case the velocity distribution has the form of a single peak situated at the maximum velocity. On the contrary, if the fluid is arrested 
in a region of volume fraction $\phi_{A}$ the distribution globally starts by a jump to a finite level ( $\left.F(0)=\phi_{A}\right)[8]$. This illustrates how the distribution function can be a fingerprint of the flow behavior.

Our results for the vertical and transversal components of the distribution function show that they are significantly different in the different regimes or for the different values of $n$ (see Figure 5) for $a=2 / 3$ : in particular for increasing Bi two steps form in the distribution, situated around $v=1$ and $v=2$, and which correspond to the growth of the two regions of almost constant velocity (see Figure 2) (orange area between two particles, and blue area in the diagonal channel). There are also some differences between the distributions obtained for $a=1 / 4$ but they are less marked, which is consistent with the observations of the velocity field (see Figure $3)$.

Finally the most striking result is that obtained for $a=1 / 9$. The distributions now appear almost identical for $\mathrm{Bi}$ ranging from 0 to 100 , and with $n$ equal to either 0.4 or 1 , both for the longitudinal and the transversal components (see Figure 6). These results are consistent with our observations of almost identical velocity fields (see Figure 4 and comments). Moreover, the arrested volume above and below the particles, which corresponds to the small step at the bottom of these curves, slightly increases with $\mathrm{Bi}$, but at its maximum only represents a volume of the order of 5\% (see Figure 2), a result which is also consistent with our above estimation from the velocity field. Finally we have a kind of breakage of the non-Newtonian character of the fluid flowing through such a geometry: the impact of the rheological behavior or flow regime on the velocity field appears negligible.

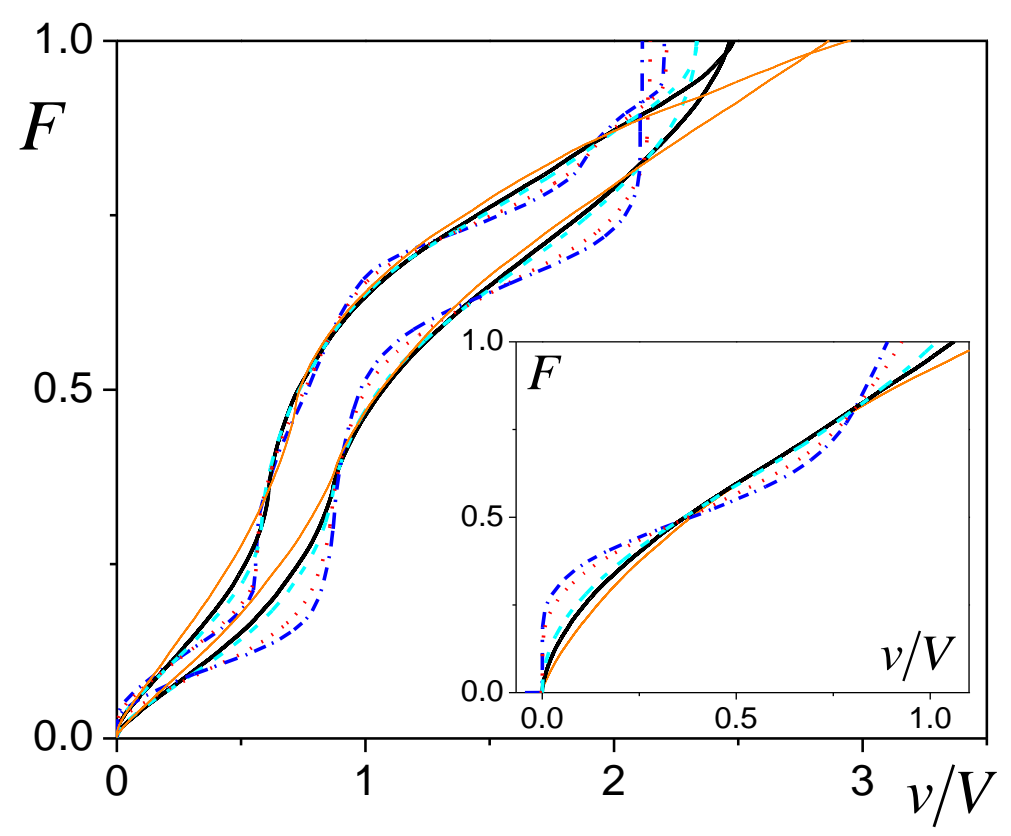

Figure 5: (Color online) Distribution function for the vertical velocity in the model porous medium for $a=1 / 4$ (upper curves) and $a=2 / 3$ (lower curves): $(n=1) \mathrm{Bi}=0$ (thin continuous orange line); $(n=0.4) \mathrm{Bi}=0$ (thick continuous black line), 1 (dashed light blue line), 10 (red dotted line), 100 (dark blue dash dot). The inset shows the distribution function for the transversal component when $a=2 / 3$. 


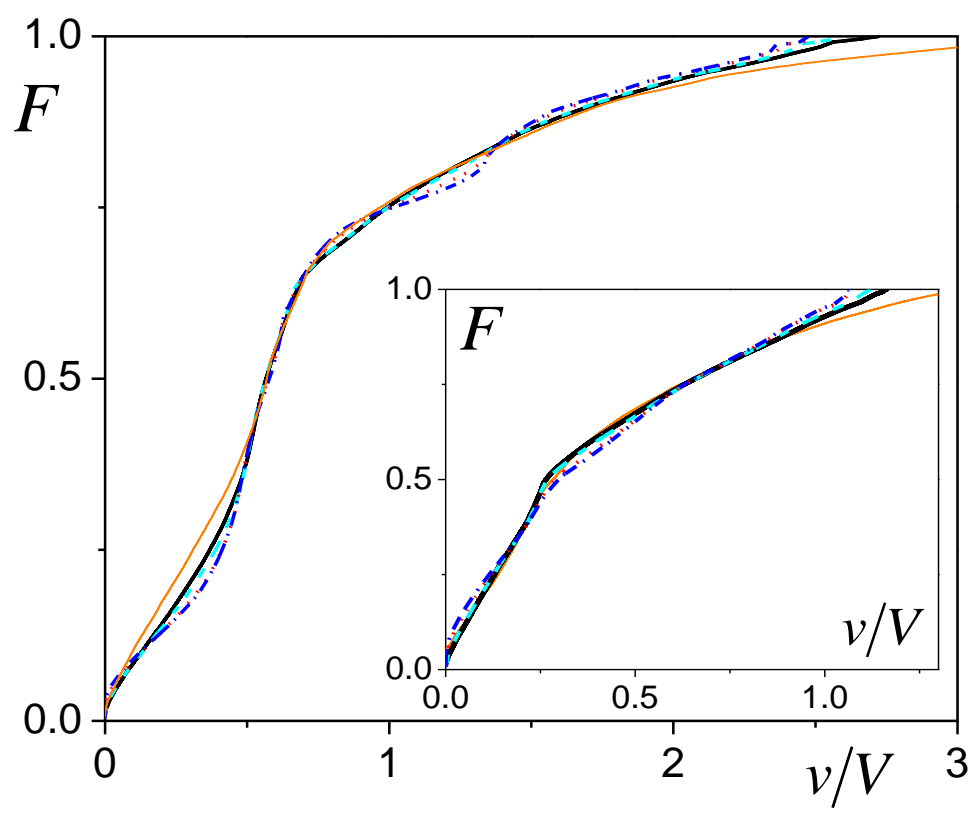

Figure 6: (Color online) Distribution function for the vertical velocity in the model porous medium for $a=1 / 9:(n=1) \mathrm{Bi}=0$ (thin continuous orange line); $(n=0.4) \mathrm{Bi}=0$ (thick continuous black line), 1 (dashed light blue line), 10 (red dotted line), 100 (dark blue dash dot). The inset shows the distribution function for the transversal component.

\section{Discussion}

We now focus on the effect of velocity field similarity observed in the case $a=1 / 9$. In view of identifying the origin of this phenomenon we can review the main characters of a porous medium as we have listed them above and see how they can have an impact on the flow characteristics. By itself the tortuosity of the geometry, i.e. the fact that the fluid has to turn around a solid region, does not seem to be able to explain the effect. Indeed, for the flow of a large volume of fluid around a single object we have strongly different flow characteristics depending on the flow regime: at high $\mathrm{Bi}$ we have a liquid region close to the object while the rest of the fluid remains in its solid regime [15], whereas for a Newtonian fluid $(\mathrm{Bi}=0)$ the flow intensity decreases slowly with the distance from the object [16]. On another side the connectivity between the pores of the porous medium does not play a role in the effect here since, due to the symmetry, it simply creates a frontier with perfect slip between the basic areas. These conclusions are further supported by the fact the velocity fields are significantly different for $a=2 / 3$ and $a=1 / 4$ whereas we already have a connectivity between the pores and a tortuosity which are very close to those in the case $a=1 / 9$.

Finally it seems that the characteristics of the porous medium which plays a major role in the breakage of the non-Newtonian character is the rapid variation of the cross-section of the flow channels. For $a=1 / 9$ the maximum distance (scaled by $d$ ) between a fluid element and a solid surface varies between 0.14 and 0.055 along the flow over a distance of 0.33 . Actually we have 
seen that if we increase the distance between the beads the effect progressively disappears: the distribution functions significantly changes when the Bingham number varies. At the same time the variation of distance between solid surfaces along the flow is smoother: for $a=1 / 4$ the maximum distance between fluid and solid varies from 0.22 to 0.125 over a distance 0.36 , and for $a=2 / 3$ it varies from 0.46 to 0.33 over a distance of 0.48 . As the value of $a$ tends to zero we have stronger reduction of the section over shorter distance. Thus the breakage of the nonNewtonian character occurs only if the variations of the cross-section of the flow channels are sufficiently rapid.

Here we suggest that through the rapid variations of the channel cross-section we more or less impose the deformation, and thus the velocity field, which explain the similarity of the velocity distribution in very different flow regimes. In order to further prove this effect let us consider the flow through the simplest geometry with a linearly varying cross-section, i.e. a trapezoidal geometry (see Figure 7). At the entrance we impose the velocity field associated with the uniform flow of the fluid at the same Bingham number in a conduit of same diameter, while the flow is free at the exit. This leads to very different velocity profiles at the entrance (see Figure $8)$.
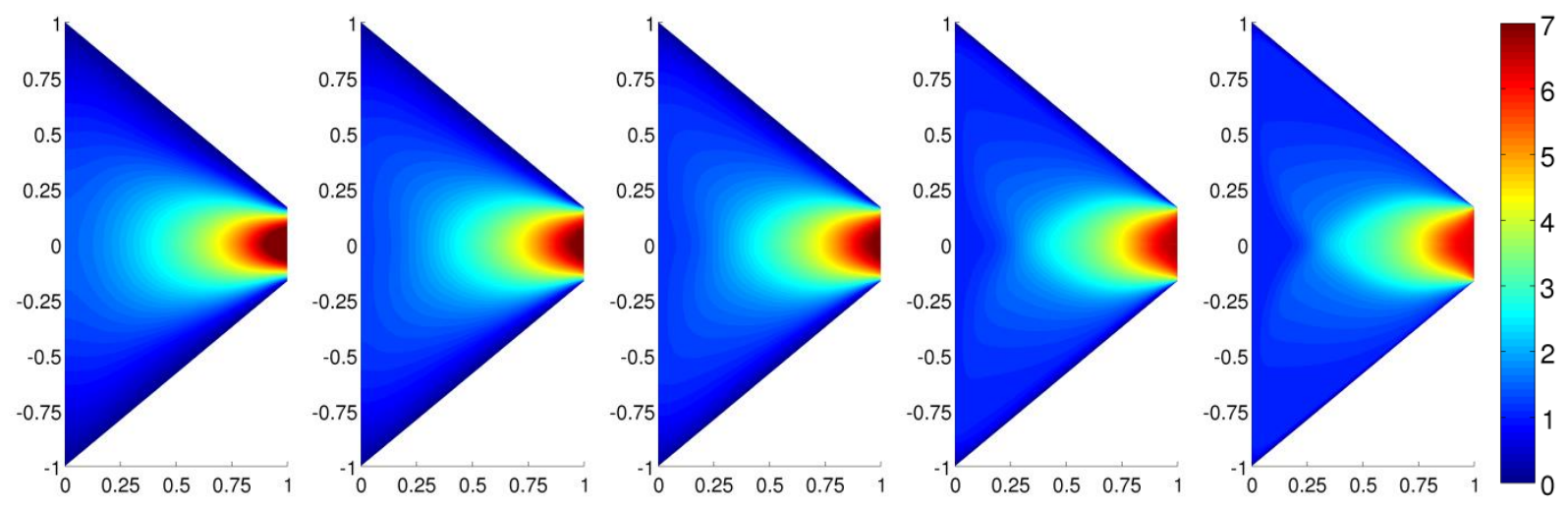

Figure 7: (Color online) Field of the velocity modulus through a trapezoidal geometry scaled by the average velocity at the entrance $(V)$ and represented in colour scales for different materials and flow regimes: (from left to right) $(n=1) \mathrm{Bi}=0 ;(n=0.4) \mathrm{Bi}=0,1,10,100$.

We find that the corresponding velocity fields in our range of $\mathrm{Bi}$ are similar at first sight (see Figure 7). In fact the significant difference around the entrance induces some difference up to about half the channel distance. Moreover the velocity fields differ close to the exit where the fluid is no longer constrained. Thus the effect of imposing the deformation may be seen after some distance of flow through this trapezoid. This may be seen more precisely from a series of horizontal velocity profiles taken at different distances from the entrance (see Figure 8): the velocity profiles are initially obviously completely different for the different $\mathrm{Bi}$ values, and as we advance in the channel they first keep this difference essentially along the wall while the rest of the fluid exhibit similar profiles for the different Bi values. Finally the difference along the wall also disappears at the approach of the exit where we have an almost unique velocity profile (with still some slight difference along the wall). Once again we see that the rapid variation of 
the channel cross-section has apparently broken the non-Newtonian character of the fluid. This effect occurs more rapidly when the deformation undergone by the fluid is larger, i.e. if the angle is larger, but it obviously disappears if the channel length or the angle are sufficiently small.

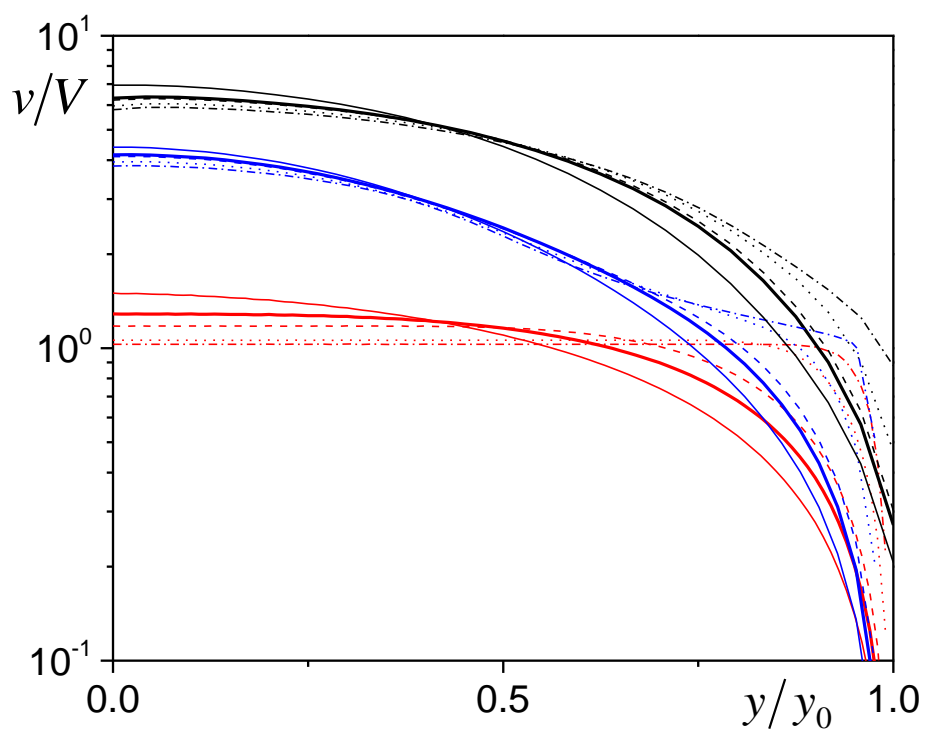

Figure 8: (Color online) Profile of the longitudinal velocity (scaled by $V$ ) as a function of the distance from the central axis scaled by the width for different distances from the entrance in the trapezoidal channel: (red, lower) $\mathrm{x}=0$, (blue, middle) $\mathrm{x}=0.7$, (black, upper) $\mathrm{x}=0.9$; and different flow regimes: $(n=1) \mathrm{Bi}=0$ (thin continuous line); $(n=0.4) \mathrm{Bi}=0$ (thick continuous line), 1 (dashed line), 10 (dotted line), 100 (dash-dot).

\section{Darcy's law for yield stress fluids}

For a yield stress fluid the usual 3D form of the constitutive equation [9], which leads to a HB model in simple shear, is: $\tau_{I I}<\tau_{c} \Rightarrow \mathbf{d}=0$ (solid regime) and $\tau_{I I}>\tau_{c} \Rightarrow \boldsymbol{\tau}=\tau_{c} \mathbf{d} / d_{I I}+2^{n} k d_{I I}{ }^{n-1} \mathbf{d}$ (liquid regime), in which $\tau$ is the deviatoric stress tensor, $\mathbf{d}$ the strain rate tensor and $d_{I I}=\sqrt{\operatorname{trd} \mathbf{d}^{2} / 2}$. The viscous dissipation inside a bead packing (of volume $\Omega$ ) is $P=\int_{\Omega} \operatorname{tr}(\mathbf{d} . \boldsymbol{\tau}) \mathrm{d} \omega$ - Introducing the constitutive equation in this expression we deduce $P=\tau_{c} \int 2 d_{I I} \mathrm{~d} \omega+k \int\left(2 d_{I I}\right)^{n+1} \mathrm{~d} \omega$, which may be rewritten as:

$P=\alpha \tau_{c} \propto \Omega(V / D)+\beta_{n} \propto \Omega(V / D)^{n+1}$

with $\alpha=(1 / \Omega \Omega) \int 2 \frac{D}{V} d_{I I} \mathrm{~d} \omega$ and $\beta_{n}=(1 / \Omega \Omega) \int\left(2 \frac{D}{V} d_{I I}\right)^{n+1} \mathrm{~d} \omega$

The above results concerning the approximately unique scaled velocity field obtained for different flow regimes lead to conclude that $(D / V) d_{I I}$ can be considered as a unique dimensionless function weakly dependent of the effective behaviour of the material or velocity and mainly depending on the structure of the porous medium. In this context $\alpha$ and $\beta_{n}$ are two 
dimensionless coefficients depending only on this distribution of shear rate intensity and on the coefficient $n$. Since the viscous dissipation may also be expressed through a macroscopic approach as $\Omega \nabla p \cdot \bar{V}=\varrho \Omega \nabla p . V$, we deduce the Darcy's law for yield stress fluids (which includes power-law and Newtonian fluids):

$\nabla p=\alpha \frac{\tau_{c}}{D}+\beta_{n} k \frac{1}{D}\left(\frac{V}{D}\right)^{n}$

The form of this law is consistent with the analysis of a series of measurements of $\nabla p$ as a function of $V$ for different yield stress fluids and pore sizes [17].

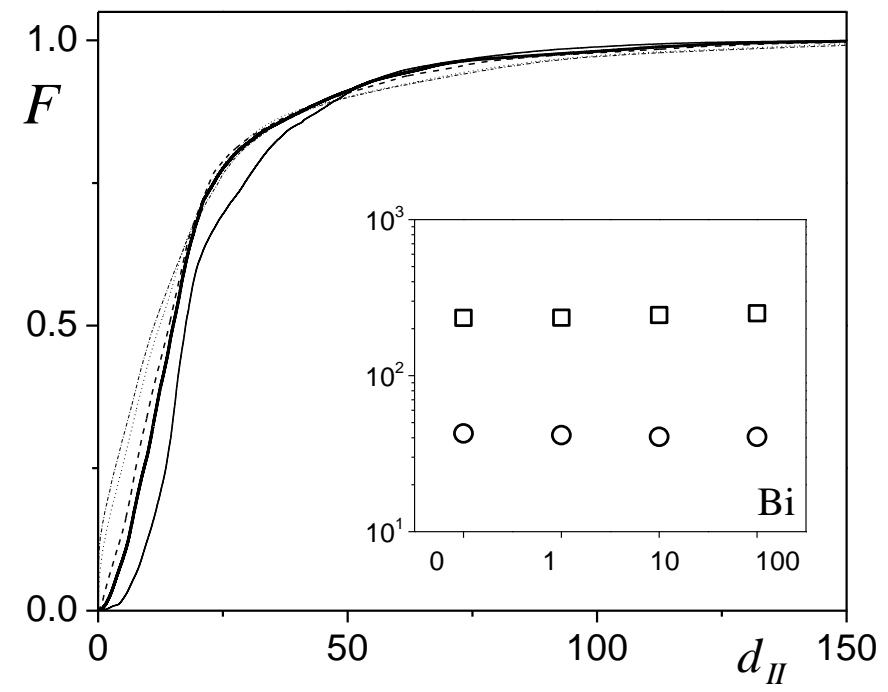

Figure 9: Distribution function for the second invariant of the strain rate tensor (scaled by $V / d$ ) for different flow regimes: $(n=1) \mathrm{Bi}=0$ (thin continuous line); $(n=0.4) \mathrm{Bi}=0$ (thick continuous line), 1 (dashed line), 10 (dotted line), 100 (dash-dot). The inset shows the corresponding values of $\alpha$ (circles) and $\beta_{n}$ (squares).

It is interesting to test the consistency of this description within the frame of the flow through the model 2D porous medium. As expected from our conclusion that the velocity is approximately independent of the flow regime the distribution function for the second invariant are very close for different Bingham number values (see Figure 9). Note however that the distribution function for the Newtonian case significantly departs from these curves at low shear rate. This tends to suggest that in this case the shear-thinning character of the fluid introduces a distinction in the flow characteristics. The two coefficients ( $\alpha$ and $\beta_{n}$ ) can be computed by integration from the results of these simulations. We find almost constant values for each of these parameters (see inset of Figure 9). Note that the value for $\alpha$ in the Newtonian case is slightly different (48.6) than the average value in the shear-thinning case with $n=0.4$ (21).

Let us know analyse the implications of these results on the description of flow through porous media. For a Newtonian fluid ( $\tau_{c}=0, k=\mu$ and $n=1$ ) the permeability $(K=\mu \bar{V} / \nabla p)$ is defined as: $K=\varepsilon D^{2} / \beta_{1}$. Measurements of $K$ for a bead packing gives $K \approx D^{2} / 1500$ [17], which implies 
that $\beta_{1} \approx 500$. Since we do not know the details of the distribution of $d_{I I}$ in the fluid we cannot immediately deduce $\alpha$ and $\beta_{n}$ from the value of $\beta_{1}$ determined from a measurement of $K$ with a Newtonian fluid. This means that the previous approaches in literature which consider that the Darcy's law for a yield stress fluid can be inferred through the Newtonian form and using the apparent viscosity of the complex fluid, are a priori erroneous.

The distribution function for the second invariant in our 2D porous medium (for the shearthinning fluid) can rather well be fitted by the function $1-\exp -x / A$ (where we now write the dimensionless second invariant $x$ ) in which $A$ is a parameter depending on the flow characteristics. Looking at the shape of the distribution function for the longitudinal velocity of the flow through a bead packing (see [8]), which can well be fitted by the same type of function, it seems probable that the distribution function for the second invariant can also well be represented by the same type of function with in that case an a priori unknown but single parameter $A$ for both the Newtonian and the shear-thinning fluids at any Bingham number value. Since now $\beta_{n}$ may be expressed as $(2 A)^{n+1} \int_{0}^{\infty}(n+1) u^{n}(\exp -u) \mathrm{d} u$ we deduce from the above experimental value for $\beta_{1}$ (500) that $A$ should be equal to 8 , thus leading to $\alpha=16$ which is not too far from the value found for simple yield stress fluids [17], i.e. Carbopol gels $(\alpha=12$ ). [Note that a smaller value for $\alpha$ (5.5) was found from a more limited and finally much less robust set of data with an emulsion]. Moreover we can now also compute $\beta_{n}$ in that case : it is equal to 50 , a value which is very close to that found from the same series of tests ( $\beta_{n}=58$, with $n=0.36)$.

\section{Conclusion}

Our results from numerical simulations show that in a flow through a complex geometry with rapid variations of cross-section the fluid properties do not play a significant role in the velocity field. These results are consistent with those obtained from NRM measurements with different fluid types flowing through granular packing. This suggests that in those flows the deformation field is more or less imposed, so that the fluid specificities are forgotten in the velocity field. Note however that the local viscous properties of the fluid still play their fundamental role in the mechanical resistance to flow.

These results also make it possible infer the general form of the Darcy's law for yield stress fluids which relies on parameters deduced from the distribution of the second invariant of the strain rate tensor. This suggests that this distribution is a fundamental characteristics of the flow through porous media, which may be considered as slightly varying with the fluid type or flow regime but varying strongly with the structure of the medium.

It remains that these results are in contradiction with those inferred from conceptual analyses [5-6] or from other types of simulations [7]. This contradiction might be due to the fact that in our approach the flow rate is clearly imposed: in the NMR experiments of [8] there is a piston pushing the fluid from upstream, and for a slow flow the fluid advances as a plug up to a very short distance from the sample entrance; in the present simulations there is no disorder and the flow rate is impose in the same through every entrance channel. On the contrary, for the previous analyses this is the pressure which is controlled, so that the fluid might have more possibilities to choose some preferential channel to flow. This difference can easily be understood from the 
simple case of a model porous medium made of two parallel ducts with different radii: if the yield stress fluid is pushed from upstream by a piston close to the cylinder entrance it will flow through both ducts; if the pressure is progressively increased the fluid will first flow through the largest duct for a critical pressure then through the second one only when the pressure will be higher than a second critical value.

\section{References}

[1] J.G. Savins, Ind. Eng. Chem., 61, 18 (1969)

[2] A.T. Unwin, Math. Eng. Ind., 5, 59 (1994)

[3] Pearson, and P.M.J. Tardy, J. Non-Newt. Fluid Mech., 102, 447 (2002)

[4] H. Pascal, F. Pascal, Int. J. Eng. Sci., 23, 571 (1985) ; T. Al-Fariss, K.L. Pinder, Can. J. Chem. Eng. 65, 391 (1987); G.C. Vradis, and A.L. Protopapas, J. Hydr. Eng., 119, 95 (1993); R.K. Dash, K.N. Mehta, G. Jayaraman, Int. J. Eng. Sci., 34, 1145 (1996) ; S. Liu, and J.H. Masliyah, Chem. Eng. Sci., 53, 1175 (1998) ; J.R.A. Pearson, and P.M.J. Tardy, J. Non-Newt. Fluid Mech., 102, 447 (2002); M. Chena, W. Rossenb, Y.C.Yortsosa, Chem. Eng. Sci., 60, 4183 (2005) ; G.G. Chase, P. Dachavijit, Rheol. Acta, 44, 495 (2005); T. Sochi, M.J. Blunt, J. Petroleum Sci. Eng., 60, 105 (2008)

[5] M.T. Balhoff, and K.E. Thompson, AIChE J., 50, 3034 (2004)

[6] M. Sahimi, AIChE J., 39, 369 (1993)

[7] L. Talon, D. Bauer, Eur. Phys. J. E, 36, 139 (2013)

[8] T. Chevalier et al., Phys. Rev. E, 89, 023002 (2014)

[9] P. Coussot, Rheometry of pastes, suspensions, and granular materials (Wiley, New York, 2005)

[10] P.M. Adler, C.J. Jacquin, J.A. Quiblier, Int. J. Multiphase Flow, 16, 691 (1990) ; F.A. Coutelieris, J.M.P.Q. Delgado, Transport Processes in Porous Media (Springer, New York, 2012)

[11] M. Bercovier, M. Engelman, J. Comput. Phys., 36, 313-326 (1980); T. C. Papanastasiou, J. Rheology, 31, 385 (1987)

[12] J. Bleyer, M. Maillard, P. de Buhan, P. Coussot, Comput. Method Appl. M., submitted (2014)

[13] M. Fortin, R. Glowinski, Augmented Lagrangian methods - Applications to the numerical solution of boundary-value problems, (Amsterdam, North-Holland, 1983); P. Saramito, N. Roquet, Comput. Method Appl. M., 190, 5391-5412, (2001); E. J. Dean, R. Glowinski, G. Guidoboni, J. Non-newt. Fluid Mech., 142, 36-62 (2007)

[14] Mosek, The Mosek optimization toolbox for Matlab manual (MOSEK ApS, Denmark, 2008)

[15] T. Chevalier, S. Rodts, X. Chateau, J. Boujlel, M. Maillard, P. Coussot, EPL, 102, 48002 (2013)

[16] H. Schlichting, Boundary Layer Theory (McGraw-Hill, New York, 1968)

[17] T. Chevalier, C. Chevalier, X. Clain, J.C. Dupla, J. Canou, S. Rodts, P. Coussot, J. Non-Newt. Fluid Mech., 195, 57 (2013) 